\title{
Effect of the specific thromboxane receptor blocking drug AH23848 in patients with angina pectoris
}

\author{
DAVID P DEBONO, * PHILIP LUMLEY, MICHAEL BEEN, * REBECCA KEERY, \\ SUSAN E INCE, DAVID F WOODINGS \\ From the *Department of Cardiology, Royal Infirmary of Edinburgh, and Glaxo Group Research Ltd, Ware, \\ Hertfordshire
}

SUMMARY The effect of the specific thromboxane receptor blocking drug AH23848 was investigated in two double blind placebo controlled studies in male patients with exercise induced angina pectoris and angiographically verified coronary lesions. In the first study cardiac pacing was performed in twenty patients after coronary angiography. Patients were then randomised into two groups and received either AH23848 (70 mg orally) or placebo. One hour later cardiac pacing was repeated. Neither treatment had any significant effect upon time to angina or the ratepressure product at the onset of chest pain in these patients. In the second study twenty male patients were randomised to seven days' treatment with AH23848 (70 mg three times a day) or placebo followed by a crossover to the other treatment for a further seven days. Clinical assessment was performed before treatment and at the end of each treatment period. There was no significant difference between the placebo and AH23848 treatment periods in exercise tolerance, the rate-pressure product at angina after exercise testing, the number of ischaemic attacks as determined from 24 hour ambulatory electrocardiograms, the number of attacks of pain, or the number of glyceryl trinitrate tablets consumed. This lack of a clinical effect with AH23848 was seen despite a profound inhibition of ex vivo platelet aggregration stimulated by the thromboxane $\mathrm{A}_{2}$-mimetic U-46619. Because in experimental animals in vivo AH23848 blocks vascular thromboxane receptors as well as platelet thromboxane receptors the lack of effect of AH23848 in cardiac pacing and exercise induced angina is unlikely to be the result of inadequate blockade of thromboxane receptors. The lack of effect of the drug is more likely to indicate that thromboxane $\mathrm{A}_{2}$ is not a factor in the aetiology of the pain experienced by these patients during exercise or cardiac pacing.

Angina pectoris is the clinical manifestation of an imbalance between myocardial oxygen demand and the supply capabilites of the coronary arterial system. Most patients with angina have considerable fixed coronary obstruction by atheroma. But there has been much interest in the possibility that dynamic coronary obstruction produced by changes in tone of the coronary vessels or temporary obstruction by platelet thrombi may also play a part. Thromboxane $A_{2}$ is released from activated platelets and is a potent inducer of platelet aggregation ${ }^{12}$ and a powerful contractile agent in both animal and

Requests for reprints to Dr David P De Bono, Department of Cardiology, The Royal Infirmary of Edinburgh, Edinburgh EH3 9YW.

Accepted for publication 4 August 1986 human coronary arteries in vitro. ${ }^{3}$ This profile of activity led several workers to propose a role for thromboxane $\mathbf{A}_{2}$ in coronary vasospasm leading to myocardial infarction, sudden cardiac death, and variant angina. ${ }^{45}$

The evidence for a role of thromboxane $A_{2}$ in human angina is, however, equivocal. There have been several reports that concentrations of thromboxane $B_{2}$, the stable degradation product of thromboxane $A_{2}$, is raised in coronary sinus blood from patients with unstable angina ${ }^{6-8}$ or during cardiac pacing in patients with angina. ${ }^{910}$ Aspirin at doses that reduced serum or urinary concentrations of thromboxane $\mathrm{B}_{2}$ by $>75 \%$ was, however, without effect upon the frequency of ischaemic incidents in patients with variant angina. ${ }^{11} 12$ In contrast two studies have recently shown aspirin to be effective in 
reducing the frequency of mycardial infarction in patients with unstable angina. ${ }^{1314}$ Equivocal results have also been reported with the thromboxane synthetase inhibitor, dazoxiben, which has been reported either to reduce ${ }^{1516}$ or to leave unaltered $^{1718}$ the ischaemic response to atrial pacing or exercise in patients with coronary artery disease.

Evidence derived from drugs that interfere with synthesis of thromboxane $A_{2}$ is, however, difficult to interpret. If the inhibition of thromboxane $A_{2}$ is achieved by inhibition of fatty acid cyclo-oxygenase the formation of other biologically active prostanoids such as prostacyclin, prostaglandins $D_{2}$ and $\mathrm{E}_{2}$ will also be inhibited, and if it is achieved by inhibition of thromboxane synthetase the production of the other products of the cyclo-oxygenase pathway may be enhanced. ${ }^{19}$ Thus in neither case it is possible to relate the end result solely to a deficiency of thromboxane $A_{2}$. The ideal compound to determine the role of thromboxane $A_{2}$ in disease states is therefore one that specifically blocks the actions of thromboxane $A_{2}$ at the level of the receptor.

AH23848 is such a drug. It has no action on cyclooxygenase, thromboxane synthetase, prostacyclin synthetase, or cyclic adenosine monophosphate phosphodiesterase enzymes. ${ }^{20}$ Over the same concentration range in vitro it inhibits platelet aggregation induced by thromboxane $\mathrm{A}_{2}$ and its stable mimetic $U-46619^{21}$ and contraction of vascular smooth muscle from various species. ${ }^{22} 23$ Most importantly, AH23848 is also equipotent in blocking platelet and vascular smooth muscle thromboxane receptors in animals in vivo 22 and would thus be expected to block both the vascular and platelet effects of thromboxane $A_{2}$ in disease states. The compound is orally active and long acting in human volunteers ${ }^{24} 25$ and is active in certain animal models of occlusive vascular disease and ischaemia. ${ }^{202627}$

We have therefore studied the effects of orally administered AH23848 in patients with exercise induced angina pectoris and angiographically verified coronary lesions in an attempt to clarify the role of thromboxane $A_{2}$ in this disease state. We chose to study this group rather than patients with unstable or "vasospastic" angina because the latter are much more difficult to define as a homogeneous group and their symptoms are often capricious and hard to reproduce. The study was conducted in two parts: as a placebo controlled double blind trial of the effect of a single dose of AH23848 on pacing induced angina and as a double blind crossover trial of $70 \mathrm{mg} \mathrm{AH} 23848$ three times daily for one week against placebo with assessment by exercise testing, ambulatory electrocardiogram monitoring, patient diaries, and glyceryl trinitrate tablet counts. In both groups of patients the magnitude of thromboxane receptor blockade produced by AH23848 was determined on platelets ex vivo so that any clinical effect of the drug could be correlated with its pharmacological effect.

\section{Patients and methods}

The studies were approved by the local advisory ethics committee. Consecutive male patients admitted for diagnostic coronary arteriography because of angina pectoris not adequately controlled by medical treatment were invited to enter the trials. Patients were excluded if they had left main coronary stenosis or had experienced a recent myocardial infarction. Their written informed consent was obtained.

\section{PACING INDUCED ANGINA}

Patients were studied at the time of coronary arteriography, which was performed by the Judkins technique $^{28}$ without heparinisation. Previous medication was discontinued at the time of hospital admission 24 hours before the study. After angiography a bipolar pacing catheter was inserted percutaneously via an arm or leg vein and positioned in the right atrial appendage. Before pacing, blood pressure was measured by sphygmomanometer cuff and auscultation. In the first study blood pressures were checked against intra-aterial blood pressures. Pacing was then started at 100 beats per minute and the rate was increased by 10 beats per minute every 20 seconds until either a rate of 140 beats per minute was reached or the maximum rate at which 1:1 atrioventricular conduction could be maintained was achieved. Pacing was continued until the patient experienced chest pain or for five minutes, whichever was the shorter. The duration of pacing, maximum pacing rate achieved, and the blood pressure at the onset of angina were recorded. The product of pacing rate and systolic blood pressure was then determined (rate-pressure product). The patient was then given $70 \mathrm{mg} \mathrm{AH} 23848$ or a placebo capsule with $100 \mathrm{ml}$ water. One hour later blood pressure was measured and pacing was then repeated and the same variables were recorded as before. The pacing electrode was then removed. The placebo group was introduced to control for the possibility that the patient might "learn" the time to angina, or alternatively be more relaxed and have a higher angina threshold at the time of the second pacing study. The long duration of action of $\mathrm{AH} 23848^{24}$ precluded any acute crossover study. 
EXERCISE INDUCED ANGINA

Patients admitted to this part of the trial all had a positive Bruce protocol excercise test ${ }^{29}$ and had undergone diagnostic coronary arteriography. They included four patients who had taken part in the first study; but at least one month had elapsed between the two studies. Before starting the study patients were clinically examined and had a 12 lead electrocardiogram, a Bruce protocol exercise test, and a 24 hour ambulatory electrocardiogram recording with two-channel frequency modulated Tracker equipment. ${ }^{30}$ Blood pressure was measured with a sphygmomanometer before and at one minute intervals during exercise testing. The electrocardiogram was monitored throughout exercise and the ratepressure product at the time of onset of angina was calculated. Patients were issued with counted glyceryl trinitrate tablets and with diaries in which to record angina attacks and record the number of glyceryl trinitrate tablets taken. Previous antianginal treatment with $\beta$ adrenoceptor antagonists, calcium channel blockers, or sustained release nitrates was not stopped nor was it altered during the trial. Patients were asked not to take aspirin or other nonsteroidal anti-inflammatory agents during and for two weeks before the trial. Patients were allocated in random order to AH23848 $70 \mathrm{mg}$ three times daily one hour before meals or to identical placebo capsules. After six days of the seven day treatment period clinical examination and exercise testing were repeated. On the day of exercise testing the time of the morning treatment was adjusted so that the exercise test took place two hours after dosing. An ambulatory electrocardiogram was recorded for 24 hours on the seventh day of the treatment period. Diaries and unused glyceryl trinitrate tablets were collected and a fresh diary and supply of glyceryl trinitrate were issued. The patient then crossed over to the alternative treatment for a further week and the tests were repeated as before. As well as comments recorded in the diaries, subjective side effects were recorded on a standard questionnaire. Blood samples for routine laboratory investigations (urea, electrolytes, liver function tests, and full blood count) were taken before treatment and after each week's treatment.

\section{STATISTICAL ANALYSIS}

Results were analysed by appropriate nonparametric statistics. For the second study we used Koch's method of applying these to a two period crossover trial. ${ }^{31}$ In addition we used methods based on Wilcoxon's rank sum statistic to compare AH23848 and placebo groups and to derive confidence intervals for each data set. ${ }^{32}$ Data for each of the measured variables were analysed separately.

\section{PLATELET AGGREGATION}

Blood was obtained from patients by venepuncture from an antecubital vein and anticoagulated with trisodium citrate. Platelet aggregation in response to the stable thromboxane $A_{2}$ mimetic U-46619 and adenosine diphosphate was monitored by a previously reported technique. ${ }^{33}$ Briefly, the number of single platelets in a $0.5 \mathrm{ml}$ volume of whole blood were counted electronically before and at intervals after addition of an aggregating agent. The peak fall in platelet count was recorded and expressed as a percentage of the control count for that volume. By adding a different concentration of aggregating agent to separate volumes of blood, aggregation concentration-effect curves were constructed (see legend to fig 1 for further experimental details).

Concentration ratios were calculated where possible on the parallel parts of the aggregation concentration-effect curves. The concentration of aggregating agent required to give $50 \%$ aggregation $\left(\mathrm{EC}_{50}\right.$ ) after treatment was divided by the mean $\mathrm{EC}_{50}$ taken from the duplicate pre-treatment control curves (see below).

\section{Pacing-induced angina}

Duplicate blood samples for control platelet aggregation studies were taken fifteen minutes apart and before angiography. A third blood sample was taken immediately after the first pacing period and a further sample one hour after treatment but before pacing. Finally, a further sample was taken immediately after the second pacing period.

\section{Exercise-induced angina}

During the pre-treatment examination of patients, but before exercise testing, duplicate blood samples for ex vivo platelet aggregation studies were taken approximately 30 minutes apart. A further sample was taken immediately after the exercise test. On the subsequent two visits single blood samples were taken before and immediately after the exercise test.

\section{DRUGS USED}

Glaxo Group Research Ltd supplied white opaque unmarked hard gelatin capsules each containing the equivalent of $35 \mathrm{mg} \mathrm{AH} 23848$ acid as the calcium salt or placebo. Trisodium citrate (BDH) was prepared as a $0.129 \mathrm{~mol} / 1$ solution in distilled water. Acetylsalicylic acid (aspirin, BDH) was dissolved in TRIS hydrochloride $(\mathrm{HCl})$ buffer $(100 \mathrm{mmol} / 1, \mathrm{pH}$ 8.5) to give a stock solution of $10 \mathrm{mg} / \mathrm{ml}$. Adenosine diphosphate (Sigma) was dissolved in Tris $\mathrm{HCl}$ buffer $(100 \mathrm{mmol} / \mathrm{l}, \mathrm{pH} 6.5)$ to give a solution strength of $1.0 \times 10^{-2} \mathrm{~mol} / 1$, dilutions were prepared in $0.9 \% \mathrm{wt} / \mathrm{vol}$ sodium chloride solution (saline). U-46619 (Glaxo Group Research) was pre- 
Table 1 Effect of AH23848 (70 mg) or placebo upon the time to angina and the rate-pressure product in patients with coronary artery disease undergoing cardiac pacing

\begin{tabular}{|c|c|c|c|c|c|}
\hline \multirow[b]{2}{*}{ Case No } & \multicolumn{2}{|l|}{ Pre-treatment } & \multicolumn{2}{|l|}{ Post-treatment } & \multirow[b]{2}{*}{$\begin{array}{l}\text { Ratio of post: } \\
\text { pretreatment } R P P\end{array}$} \\
\hline & $\begin{array}{l}\text { Time to angina } \\
\text { (min) }\end{array}$ & $R P P$ & $\begin{array}{l}\text { Time to angina } \\
\text { (min) }\end{array}$ & $R P P$ & \\
\hline \multicolumn{6}{|c|}{ AH23848: } \\
\hline 01 & $5 \cdot 0$ & 19800 & 3.5 & 20400 & 1.03 \\
\hline 03 & $5 \cdot 0$ & 13800 & $5 \cdot 0$ & 14950 & 1.08 \\
\hline 06 & $5 \cdot 0$ & 12350 & $5 \cdot 0$ & 12610 & 1.02 \\
\hline 07 & 1.5 & 14300 & 1.5 & 15950 & $1 \cdot 12$ \\
\hline 10 & $5 \cdot 0$ & 12100 & $5 \cdot 0$ & 13200 & 1.09 \\
\hline 12 & 3.0 & 15600 & $5 \cdot 0$ & 21450 & $1 \cdot 38$ \\
\hline 13 & $5 \cdot 0$ & 14400 & $5 \cdot 0$ & 13200 & 0.92 \\
\hline 14 & $5 \cdot 0$ & 14300 & $5 \cdot 0$ & 18200 & $1 \cdot 27$ \\
\hline 19 & $3 \cdot 5$ & 14375 & $5 \cdot 0$ & 13750 & 0.96 \\
\hline 20 & 1.5 & 15600 & $2 \cdot 5$ & 13200 & 0.85 \\
\hline \multicolumn{6}{|l|}{ Placebo: } \\
\hline 02 & $5 \cdot 0$ & 13800 & $5 \cdot 0$ & 8970 & 0.65 \\
\hline 04 & $5 \cdot 0$ & 14300 & $5 \cdot 0$ & 11050 & 0.77 \\
\hline 05 & $5 \cdot 0$ & 11760 & $5 \cdot 0$ & 11400 & 0.97 \\
\hline 08 & $5 \cdot 5^{\star}$ & 9900 & $5 \cdot 0$ & 11500 & $1 \cdot 17$ \\
\hline 09 & $5 \cdot 0$ & 10925 & $5 \cdot 0$ & 11270 & 1.03 \\
\hline 11 & $5 \cdot 0$ & 18900 & $5 \cdot 0$ & 16200 & 0.86 \\
\hline 15 & $5 \cdot 0$ & 14175 & $5 \cdot 0$ & 15120 & 1.07 \\
\hline 16 & $3 \cdot 5$ & 11700 & $3 \cdot 0$ & 14300 & $1 \cdot 22$ \\
\hline 17 & 3.0 & 14300 & $4 \cdot 5$ & 17550 & $1 \cdot 23$ \\
\hline 18 & 1.5 & 20000 & $4 \cdot 5$ & 13750 & 0.69 \\
\hline
\end{tabular}

RPP, rate-pressure product.

$\star$ Pacing continued for 5.5 minutes in this patient.

pared as a $1.0 \times 10^{-2} \mathrm{~mol} / \mathrm{l}$ solution in $1 \% \mathrm{wt} / \mathrm{vol}$ sodium bicarbonate in saline; dilutions were prepared in saline. All drug solutions were stored on ice during use. All concentrations of drugs refer to whole blood.

\section{Results}

\section{PACING-INDUCED ANGINA}

Twenty male patients with stable angina were entered into this study. Of these patients $13 \mathrm{had}$ three vessel disease, four had two vessel disease, and three had single vessel disease. Fourteen patients subsequently went on to have coronary artery bypass graft surgery. The age distributions were similar for the two groups of patients, the median and range being $54(40-59)$ years for placebo and 56 (41-65) years for AH23848. There was no significant difference between the groups in precatheter exercise tolerance, coronary artery score, or subsequent requirement for coronary surgery.

In both groups of patients treatment had no effect on the time to angina, the mean (range) in minutes before and after placebo being $4.4(1.5-5.5)$ and 4.7 (3.0-5.0) respectively compared with $4.0(1.5-5.0)$ and 4.3 $(1.5-5.0)$ before and after AH23848. The rate-pressure product was similarly unaffected by either treatment (table 1). Diastolic blood pressure at the onset of angina was significantly higher in patients receiving $\mathrm{AH} 23848$ than in patients receiving placebo (median difference $(95 \%$ confidence interval) $13(5,30) \mathrm{mm} \mathrm{Hg})$. In contrast, diastolic pressure was not higher in the AH23848 group before pacing nor was such a difference seen at angina during the pre-treatment pacing. In addition, no such difference in systolic blood pressure was seen between the two groups of patients either before pacing or at angina. The reason for the slightly higher diastolic blood pressure at angina after AH23848 treatment is not known.

Despite the apparent lack of effect of AH23848 upon the variables assessed during pacing, this agent did have a profound effect upon U-46619-induced ex vivo platelet aggregation in blood samples taken one hour after drug treatment. In all of the patients given AH23848 a profound rightward displacement of the U-46619 concentration effect curve was seen. In only one patient was a U-46619 concentration ratio of $<50$ obtained (range 19-251); in contrast platelet aggregation induced by adenosine diphosphate was not affected by AH23848 treatment. Placebo treatment was also without effect upon aggregation in response to both aggregating agents. Figure 1 shows the mean aggregation concentrationeffect curves for the two groups.

During this study blood samples were taken immediately after the end of cardiac pacing. These were taken to determine whether pacing per se pro- 


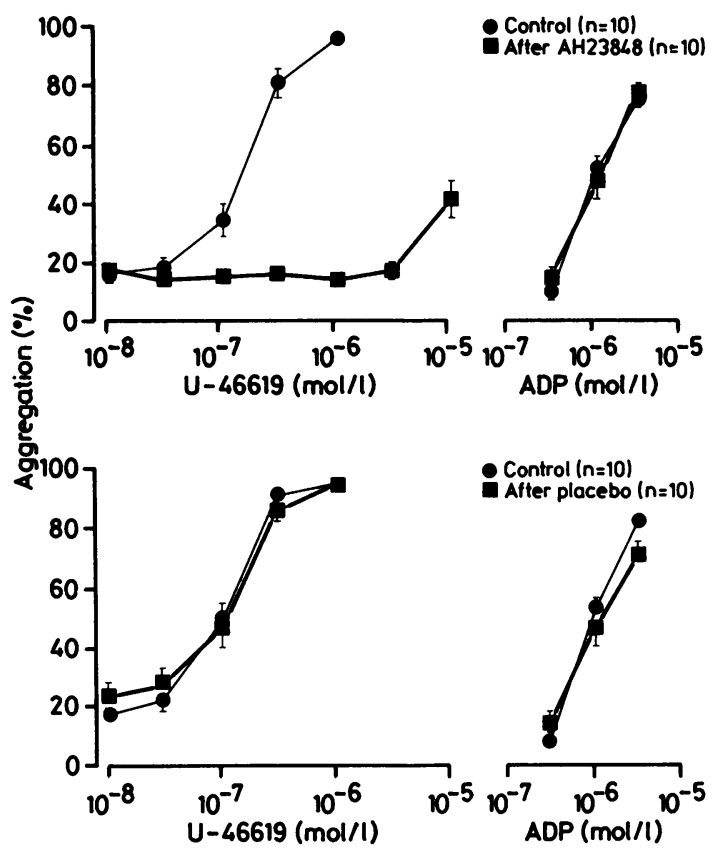

Fig 1 The effect (mean (SE)) of a single oral dose of either $A H 23848$ ( $70 \mathrm{mg}$ ) (upper panel) or placebo (lower panel) upon platelet aggregation induced by U-46619 and adenosine diphosphate ( $A D P$ ) ex vivo in whole blood from patients with angina pectoris undergoing cardiac pacing. Blood samples $(4.5 \mathrm{ml})$ were obtained by venepuncture and anticoagulated with trisodium citrate (final concentration $12.9 \mathrm{mmol} / \mathrm{l}$ ). Volumes of whole blood were then incubated at $37^{\circ} \mathrm{C}$ in a shaking water bath while aggregation studies were performed. Aspirin (2.0 mmol $/ \mathrm{l}$ ) was added to blood volumes to prevent the formation of endogenous prostaglandins during the aggregation process. Aggregation studies were started as rapidly as possible, usually 10-15 minutes after venepuncture. Platelet counts were determined before and 10, 20, and occasionally 30 minutes after addition of $U-46619$, the peak fall in platelet count usually occurring at 10 minutes in control and placebo blood samples but up to 30 minutes after AH23848 treatment. Platelet counts were determined before and one minute after àddition of adenosine diphosphate ( $A D P$ ); this has previously been shown to represent the time of peak aggregation. ${ }^{33}$ The control curves represent the second of the two preliminary U-46619 and adenosine diphosphate curves.

duced any change in platelet sensitivity and whether treatment with a thromboxane receptor blocking drug would modify any such change. Comparison of the $\mathrm{EC}_{50}$ values of the control concentration effect curves in response to both $U-46619$ and adenosine diphosphate with those from curves obtained after the first pacing period from the twenty patients showed no consistent change in platelet sensitivity that could be attributed to pacing.

Few side effects were noted in this study. One patient became dizzy and hypotensive after taking placebo, one experienced nausea 30 minutes after taking AH23848, and a third patient experienced chest pain not typical of angina one hour after taking AH23848 but before the second pacing.

\section{EXERCISE-INDUCED ANGINA}

Twenty male patients including four who had taken part in the pacing-induced angina study, entered the study. Of these patients 13 had three vessel disease, four had two vessel disease, and two had single vessel disease (the remaining patient was withdrawn, see below). Eleven patients subsequently went on to have coronary artery bypass graft surgery. Two patients did not complete the study: patient 3 was called in for coronary artery surgery after completing only one week of the study and patient 10 was withdrawn before receiving active treatment because of a sudden deterioration in exercise tolerance. Patient 1 inadvertently took only half the dose of AH23848 and was therefore excluded from the analysis. Of the remaining 17 patients platelet aggregation studies indicated non-compliance in three patients (numbers 11,13, and 17, see below) and in patient 8 aggregation studies were incomplete. Results were therefore analysed twice, both including these patients $(n=17)$ and excluding them $(n=$ 13). Both analyses gave the same result.

Table 2 summarises the results. There was no significant difference in exercise tolerance, number of ischaemic attacks (defined as a depression of the $J$ junction by at least $1 \mathrm{mV}$ followed by an ST segment depression sloping downwards detected by ambulatory electrocardiogram monitoring), number of attacks of pain, or number of glyceryl trinitrate tablets consumed between the placebo and AH23848 treatment periods. In addition we found no significant difference between the two treatments in the rate-pressure product at angina after exercise testing nor any effect on blood pressure.

As in the first study, patients receiving AH23848 for six days generally showed a large rightward displacement in the U-46619 aggregation concentration-effect curve. We saw no effect upon adenosine diphosphate induced aggregation. Figure 2 summarises the data for all patients. Patients who received AH23848 as the first treatment showed no residual blocking effect of the drug on day 6 of the subsequent placebo treatment. Patients 11, 13, and 17 unexpectedly displayed low U-46619 concentration ratios after AH23848 (1.7, 0.7, and $5 \cdot 2$ respectively) and were deemed to be non-compliant. This apparent lack of effect after AH23848 treatment was also seen in the blood samples from these 
Table 2 Effect of AH23848 or placebo for seven days upon various indices of ischaemia in patients with angina pectoris undergoing exercise stress testing

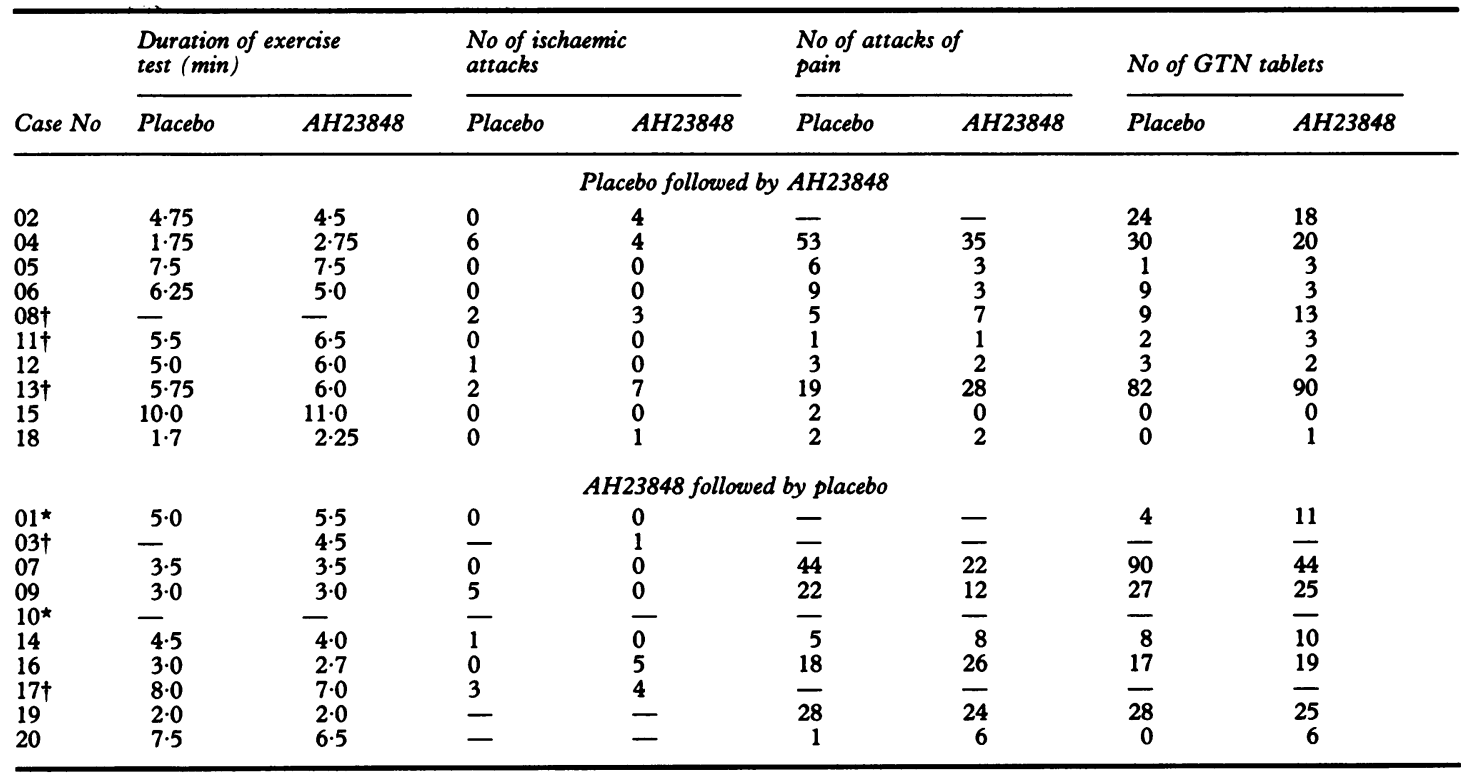

*Patients excluded from analysis (see text).

†Analyses performed with and without these patients (see text).

-Measurement not carried out.

patients after the exercise testing, thus ruling out an erroneous platelet aggregation determination. A ratio of 10 was chosen as the limit below which patient non-compliance was assumed. This value was based upon those obtained from patients given AH23848 in the first study and our experience in healthy volunteers taking this dosage regimen. ${ }^{24} 25$

Placebo did not have a pronounced effect on aggregation in response to U-46619 or adenosine diphosphate, except in patient 17 in whom platelet aggregation in response to the thromboxane $\mathbf{A}_{2}$ mimetic appeared to be antagonised more by placebo treatment than by AH23848. This was one of the patients deemed to be non-compliant.

The effect of exercise per se upon platelet aggregation was also studied. In blood samples taken immediately after the pre-treatment exercise test there was no consistent change in platelet sensitivity to either U-46619 or adenosine diphosphate.

In the second study two patients experienced malaise and one patient described diffuse muscle aches while taking placebo; one patient had heartburn, nausea, and vomiting while taking AH23848 and one patient reported feeling very tired while taking the active drug. No clinically significant changes in laboratory values were noted.

\section{Discussion}

The present study was designed to test the hypothesis that thromboxane $A_{2}$ has a role in angina induced by cardiac pacing and exercise in patients with angiographically confirmed coronary artery disease and therefore whether a thromboxane $A_{2}$ receptor blocking drug would be therapeutically useful in this condition.

Evidence for an involvement of thromboxane $\mathbf{A}_{2}$ in various forms of angina has been largely circumstantial. Thus for example raised concentrations of thromboxane $\mathbf{B}_{2}$ have been measured in coronary sinus blood during pacing in angina patients. ${ }^{91017}$ However, sampling procedures themselves, especially withdrawal of blood through long indwelling catheters, may induce platelet activation and thromboxane $\mathbf{A}_{2}$ release. ${ }^{34}$ If platelets are more reactive after cardiac pacing than before pacing, different degrees of activation and hence thromboxane $A_{2}$ release may occur with sampling. These raised concentrations would not then necessarily imply a pathophysiological role for thromboxane $\mathbf{A}_{2}$ per se.

Evidence from drug studies is also difficult to interpret. Negative studies with cyclo-oxygenase 


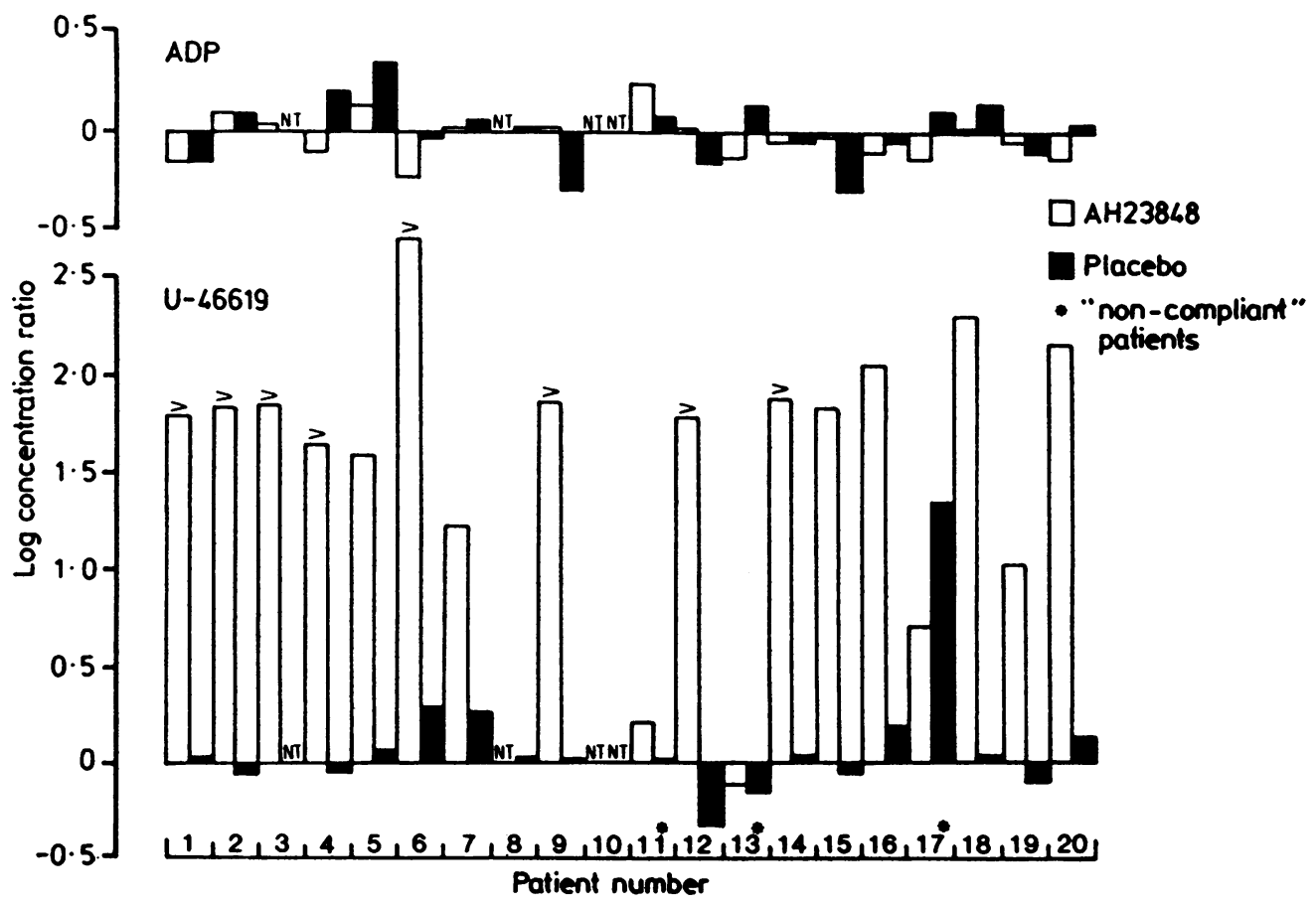

Fig 2 Effect of AH23848 and placebo upon platelet aggregation ex vivo in angina patients undergoing exercise tolerance testing. Histograms represent concentration ratios for $U-46619$ and adenosine diphosphate after treatment with either AH23848 or placebo. Columns designated > represent minimum estimates of U-46619 concentration ratio values when $50 \%$ aggregation to $U-46619$ was not achieved in the post-AH23848 blood samples. In these instances the $U-46619 E_{50}$ value during treatment was assigned a value of $>1.0 \times 10^{-5}$ mol/l, the highest concentration of aggregating agent used. In patients 11 and 13 there was no antagonism of U-46619-induced aggregation after AH23848 treatment and in patient 17 placebo treatment appeared to be more effective than drug treatment. This profile was not expected from previous experience with the drug. NT, not tested.

inhibitors such as aspirin in pacing-induced angina ${ }^{16}$ may be a consequence of the reduction in prostacyclin and platelet-derived prostaglandin $D_{2}$ concentrations rather than reduction in thromboxane $\mathrm{A}_{2}$ itself. The balance between prostacyclin and thromboxane $A_{2}$ has been shown to be important in animal models of cardiac ischaemia. ${ }^{35}$ In contrast, positive findings with the thromboxane synthetase inhibitor dazoxiben ${ }^{1516}$ could equally well reflect the beneficial diversion of endogenous prostaglandin endoperoxides to prostacyclin rather than to a reduction in thromboxane $A_{2}$ concentrations.

As has been pointed out previously ${ }^{20}$ definitive evidence for the action of thromboxane $A_{2}$ in a disease state depends upon the use of a highly specific thromboxane $A_{2}$ receptor blocking drug. Such a drug would only block the actions of thromboxane $\mathrm{A}_{2}$ and leave untouched the production of thromboxane $\mathrm{A}_{2}$ and prostacyclin. AH23848 is a specific and potent thromboxane $A_{2}$ receptor blocking drug that has no effect upon cyclo-oxygenase, thromboxane synthetase, or prostacyclin synthetase. ${ }^{20}$ In addition it is non-selective, being approximately equipotent in blocking thromboxane $\mathrm{A}_{2}$ receptors present on both the platelet and vascular smooth muscle. This profile has been observed both in isolated tissues and in vivo in laboratory animals. ${ }^{22}$ AH23848 would therefore be expected to block local vasoconstriction as well as platelet aggregation induced by thromboxane $A_{2}$ in vivo.

In the present study AH23848 did not have any clinically beneficial effect on angina pectoris after pacing or exercise in patients with angiographically confirmed coronary artery disease. Platelet aggregation in response to the thromboxane $A_{2}$ mimetic U-46619, however, was substantially inhibited by AH23848 at the time of pacing or exercise. Sufficient thromboxane receptor blockade was achieved to produce an approximately 100-fold rightward displacement of the U-46619 platelet aggregation concentration-effect curves. In contrast with the 
effect upon U-46619, adenosine diphosphate induced aggregation, which occurs independently of thromboxane $A_{2}$ under the conditions of this study, was unaffected. This profile of action would be expected of a specifically acting receptor blocking drug such as AH23848. Thus in the patients studied a very large level of platelet thromboxane $A_{2}$ receptor blockade was apparent yet no effect on time to pain was seen in either study. As mentioned above, in experimental studies AH23848 also produces a similar degree of blockade of vascular thromboxane receptors to that produced on the platelet thromboxane receptor. While the pharmacodynamic profile of the actions of this drug upon vascular thromboxane receptor in man has not been studied, both pacing and exercise testing were performed within 1-2 hours of AH23848 administration. Thus it is presumed that a large degree of blockade of the vascular thromboxane receptor was also achieved in these patients. These results are therefore strong evidence for the lack of effect of thromboxane $A_{2}$ in angina, at least in the patients we studied and under the circumstances of this study.

In the exercise study several patients were excluded from analysis because there was no antagonism of the U-46619 induced aggregation responses after AH23848. We believed this action to be justified because our experience with the drug in volunteers and patients ${ }^{242536}$ had never shown such a lack of effect. Two analyses performed after inclusion or exclusion of the "non-compliant" patients were both negative.

We believe that the measurement of antagonism of the aggregatory action of U-46619 by AH23848 was essential in the present study so that we could reach valid conclusions about the actions of thromboxane $A_{2}$ in these patients. Does this level of thromboxane receptor blockade have any clinical relevance, however? Other studies suggest that it does. Thus in patients with mature aorto-bifemoral arterial Dacron grafts AH23848 appeared to reduce deposition of platelets onto the grafts. ${ }^{36}$ In the deposition study AH23848 was administered to patients for a similar period (nine days) to that used in the present exercise study and produced a similar level of antagonism of U-46619 induced platelet aggregation. Thus the lack of effect of AH23848 in the present study is unlikely to be caused by an inadequate level of thromboxane receptor blockade.

It could, however, also be argued that since several patients in our study were taking $\beta$ adrenoceptor antagonists, calcium channel blockers, or nitrates any minor antianginal effect of AH23848 might be overshadowed. Our ethical advisory committee was unhappy about stopping all medication in patients with known severe angina for the trial of an agent of uproven efficacy. It might be expected that an antianginal agent with a completely different mode of action may have an additive or synergistic effect, however, as has been demonstrated for $\beta$ adrenoceptor antagonists and calcium channel blockers. The present study does not necessarily contradict observations of increased thromboxane $\mathrm{A}_{2}$ production in angina, but suggests this is a secondary rather than a primary phenomenon. Nor does it exclude a long term role for platelet activation in extending or perpetuating coronary arterial damage.

Finally, we chose to study patients with exercise induced rather than unstable or vasospastic angina, for the reasons stated in the Introduction and also because we wished to become more familiar with the clinical properties of AH23848 before using it in patients in an unstable state. A priori, vasospastic angina might be more likely to respond to a thromboxane receptor blocking drug. In fact the recent studies with aspirin ${ }^{1314}$ have provided evidence for the involvement of thromboxane $A_{2}$ in this condition. Specific thromboxane receptor blocking drugs like AH23848, by sparing vascular prostacyclin and platelet derived prostaglandin $D_{2}$, may prove to be more effective than aspirin.

In conclusion, AH23848 was well tolerated but was without effect upon the indices of ischaemia in patients with pacing and exercise induced angina pectoris despite producing a considerable blockade of the platelet thromboxane $A_{2}$ receptor. The results of the present study strongly suggest, therefore, that thromboxane $A_{2}$ is not important in severe angina pectoris. AH23848 is a valuable drug, and drugs like it, in addition to resolving the controversy about the involvement of thromboxane $A_{2}$ in various diseases, may also prove to be valuable therapeutic agents.

\section{References}

1 Hamberg M, Svensson J, Samuelson B. Thromboxanes: a new group of biologically active compounds derived from prostaglandin endoperoxides. Proc Natl Acad Sci USA 1975;72:2994-8.

2 Bhagwat SS, Hamann PR, Still WC, Bunting S, Fitzpatrick FA. Synthesis and structure of the platelet aggregation factor thromboxane $A_{2}$. Nature 1985;315:511-3.

3 Kulkarni PS, Wang H-H. Pharmacology of human coronary arteries [Abstract]. Pharmacologist 1970;22:217.

4 Ellis EF, Oelz O, Roberts LJ, et al. Coronary arterial smooth muscle contraction by a substance released from platelets: evidence that it is thromboxane $\mathbf{A}_{2}$. Science 1976;193:1135-7.

5 Needleman P, Kulkarni PS, Raz A. Coronary tone modulation: formation and actions of prostaglandins, 
endoperoxides and thromboxanes. Science 1977;195: 409-12.

6 Lewy RI, Smith JB, Silver MJ, Saia J, Walinsky P, Weiner L. Detection of thromboxane $B_{2}$ in peripheral blood of patients with Prinzmetal's angina. Prostaglandins and Medicine 1979;2:243-8.

7 Hirsch PD, Hillis LD, Campbell WB, Firth BG, Willerson JT. Release of prostaglandins and thromboxane into the coronary circulation in patients with ischemic heart disease. $N$ Engl f Med 1981;304: 685-91.

8 De Boer AC, Turpie AG, Butt RW, Johnston RV, Genton E. Platelet release and thromboxane synthesis in symptomatic coronary artery disease. Circulation 1982;66:327-33.

9 Lewy RL, Weiner L, Walinsky P, Lefer AM, Silver MJ, Smith JB. Thromboxane release during pacinginduced angina pectoris. Possible vasoconstrictor influence on coronary vasculature. Circulation 1980;61:1165-71.

10 Tada $M$, Kuzuya $T$, Inoue $M$, et al. Evaluation of thromboxane $B_{2}$ levels in patients with classic and variant angina pectoris. Circulation 1981;64:1107-15.

11 Robertson RM, Robertson D, Roberts LJ, et al. Thromboxane $A_{2}$ in vasotonic angina pectoris. Evidence from direct measurements and inhibitor trials. N Engl f Med 1981;304:998-1003.

12 Chierchia S, Caterina R, Crea F, Patrono C, Maseri A. Failure of thromboxane $A_{2}$ blockade to prevent attacks of vasospastic angina. Circulation 1982; 66:702-5.

13 Lewis HD, Davis JW, Archibald DG, et al. Protective effect of aspirin against acute myocardial infarction and death in men with unstable angina. $N$ Engl $f$ Med 1983;309:396-403.

14 Cairns J, Gent M, Singer J, et al. A study of aspirin and/or sulfinpyrazone in unstable angina [Abstract]. Circulation 1984;70:415.

15 Hutton I, Tweddel AC, Rankin AC, Walker ID, Davidson JF. Effects of dazoxiben on transcardiac thromboxane levels and haemodynamics in coronary heart disease. Br f Clin Pharmacol 1983;15:79S-82S.

16 Thaulow E, Dale J, Myhre E. Effects of a selective thromboxane synthetase inhibitor, dazoxiben, and of acetylsalicylic acid on myocardial ischaemia in patients with coronary artery disease. Am $\mathcal{F}$ Cardiol 1984;53:1255-8.

17 Kiff PS, Bergman G, Atkinson L, Jewitt DE, Westwick J, Kakkar VV. Haemodynamic and metabolic effects of dazoxiben at rest and during atrial pacing. $\mathrm{Br} \mathcal{F}$ Clin Pharmacol 1983;15:73S-7S.

18 Rueben SR, Kuan P, Cairns J, Gyde OH. Effects of dazoxiben on exercise performance in chronic stable angina. $\mathrm{Br} \mathcal{F}$ Clin Pharmacol 1983;15:83S-6S.

19 Vermylen J, Defreyn G, Carreras LO, Machin SJ, van Schaeren J, Verstraete $M$. Thromboxane synthetase inhibition as antithrombotic strategy. Lancet 1981 ; i:1073-5.

20 Brittain RT, Boutal L, Carter MC, et al. AH23848: a thromboxane receptor blocking drug which can clarify tie pathophysiological role of thromboxane A2. Circulation 1985;72:1208-18.
21 Coleman RA, Humphrey PPA, Kennedy I, Levy GP, Lumley P. Comparison of the actions of U-46619, a prostaglandin $\mathrm{H}_{2}$-analogue, with those of prostaglandin $\mathrm{H}_{2}$ and thromboxane $\mathrm{A}_{2}$ on some isolated smooth muscle preparations. $\mathrm{Br} \mathcal{F}$ Pharmacol 1981;73:773-8.

22 Humphrey PPA, Lumley P. The effects of AH23848, a novel thromboxane receptor blocking drug, on platelets and vascular smooth muscle [Abstract]. Br f Pharmacol 1984;83:378P.

23 Brittain RT, Coleman RA, Collington EW, et al. AH23848: a potent, selective thromboxane receptor blocking drug on smooth muscle [Abstract]. $\mathrm{Br} \mathcal{F}$ Pharmacol 1984;83:377P.

24 Thomas M, Lumley P, Hornby EJ. A study to investigate the effects of a novel thromboxane receptor blocking drug $\mathrm{AH} 23848$ on platelet aggregation ex vivo in man [Abstract]. $\mathrm{Br} \boldsymbol{F}$ Clin Pharmacol 1985;19:123P-4P.

25 Thomas M, Lumley P, Fowler P. Effect of repeat dosing with the thromboxane receptor blocking drug AH23848 on platelet aggregation ex vivo in man [Abstract]. Br f Clin Pharmacol 1985;20:543P-4P.

26 Coker SJ, Parratt JR. AH23848, a thromboxane antagonist, suppresses ischaemia and reperfusion-induced arrhythmias in anaesthetised greyhounds. $\mathrm{Br} \mathcal{F}$ Pharmacol 1985;86:259-64.

27 Boutal LE, Foster MR, Geisow HP, Skidmore IF. Inhibition of 111-indium labelled platelet deposition by aspirin, prostacyclin and the thromboxane antagonist, AH23848 [Abstract]. Br $\mathcal{f}$ Pharmacol 1985;86:802P.

28 Judkin MP. Selective coronary arteriography I. Percutaneous transfemoral technique. Radiology 1967;89:815-21.

29 Bruce RA, McDonough JR. Stress testing in screening for cardiovascular disease. Bull NY Acad Med 1969;45:1288-305.

30 Kunkes SH, Pichard A, Meller SJ, Gorlin R. Herman MV, Kupersmith J. Use of the ambulatory electrocardiogram to diagnose coronary disease. $\mathcal{f}$ Electrocardiol 1980;13:341-6.

31 Koch GG. The use of non-parametric methods in the statistical analysis of the two period changeover design. Biometrics 1972;28:577-84.

32 Hollander M, Wolfe DA. Non-parametric statistical methods. New York: Wiley, 1973:68-75.

33 Lumley P, Humphrey PPA. A method for quantifying platelet aggregation and analysing drug-receptor interactions on platelets in whole blood in vitro. f Pharmacol Methods 1981;6:153-66.

34 Fitzgerald GA, Pedersen AK, Patrono C. Analysis of prostacyclin and thromboxane biosynthesis in cardiovascular disease. Circulation 1983;67:1174-7.

35 Coker SJ, Parratt JR, Ledingham I McA, Zeitlin IJ. Thromboxane and prostacyclin release from ischaemic myocardium in relation to arrhythmias. Nature 1981;291:323-4.

36 Lane IF, Irwin JTC, Jennings SA, Poskitt KR, Greenhalgh RM, McCollum CN. A specific thromboxane $A_{2}$-antagonist evaluated in vascular graft patients [Abstract]. Br F Surg 1984;71:903. 